\title{
Inelastic $x$-ray scattering study of plasmon dispersions in solid and liquid $\mathrm{Rb}$
}

\section{AUTHOR(S):}

Kimura, K.; Matsuda, K.; Hiraoka, N.; Fukumaru, T.; Kajihara, Y.; Inui, M.; Yao, M.

\section{CITATION:}

Kimura, K. ...[et al]. Inelastic x-ray scattering study of plasmon dispersions in solid and liquid Rb. Physical Review B 2014, 89(1):

014206.

\section{ISSUE DATE:}

2014-01

URL:

http://hdl.handle.net/2433/193244

RIGHT:

C)2014 American Physical Society. 
PHYSICAL REVIEW B 89, 014206 (2014)

\title{
Inelastic $\mathbf{x}$-ray scattering study of plasmon dispersions in solid and liquid $\mathbf{R b}$
}

\author{
K. Kimura, ${ }^{1}$ K. Matsuda, ${ }^{1, *}$ N. Hiraoka, ${ }^{2}$ T. Fukumaru, ${ }^{1}$ Y. Kajihara, ${ }^{3}$ M. Inui, ${ }^{3}$ and M. Yao ${ }^{1}$ \\ ${ }^{1}$ Graduate School of Science, Kyoto University, Kyoto 606-8502, Japan \\ ${ }^{2}$ National Synchrotron Radiation Research Center, Hsinchu, 30076, Taiwan \\ ${ }^{3}$ Graduate School of Integrated Arts and Sciences, Hiroshima University, Higashi Hiroshima 739-8521, Japan
}

(Received 10 April 2013; revised manuscript received 14 January 2014; published 29 January 2014)

\begin{abstract}
The plasmon dispersion relations in solid and liquid $\mathrm{Rb}$ were determined by inelastic $\mathrm{x}$-ray scattering techniques. In liquid $\mathrm{Rb}$, the plasmon energy increases with the momentum transfer $q$, whereas in solid $\mathrm{Rb}$, the dispersion curve exhibits a cusplike shape. We also found that the plasmon linewidth in liquid Rb is narrower than that in solid $\mathrm{Rb}$ near $q=0$. These features suggest that the conduction electrons in liquid $\mathrm{Rb}$ are more suitably described by the electron gas model than in solid $\mathrm{Rb}$, since the electron gas model predicts a smooth increase in the plasmon energy with $q$ and the infinite lifetime at $q=0$. The origins of the observed variations in the plasmon energy and the linewidth upon melting are investigated with the aid of theoretical calculations.
\end{abstract}

DOI: 10.1103/PhysRevB.89.014206

PACS number(s): 71.22.+i, 71.45.Gm

\section{INTRODUCTION}

The behavior of conduction electrons in alkali metals is basically well described by the electron gas model, which is manifested, for example, in the almost spherical shape of the Fermi surface. However, the electron energy loss spectroscopy (EELS) experiments for solid alkali metals ( $\mathrm{Na}, \mathrm{K}, \mathrm{Rb}$, and $\mathrm{Cs)}$ [1] revealed that the plasmon dispersions in $\mathrm{Rb}$ and Cs deviate significantly from the interacting electron gas model [2], which predicts that the plasmon energy smoothly increases with the momentum transfer $q$. The experimentally obtained plasmon energy in solid Rb only slightly increases as $q$ becomes larger and then starts to decrease around $q \sim 0.60 \AA^{-1}$, showing the "cusplike" behavior. In the case of solid Cs, even a negative dispersion is observed.

Motivated by the EELS measurements of alkali metals, many theoretical works have been carried out [3-10]. Several studies $[3,5,6]$ which treated the electron-electron interactions in a more sophisticated manner were performed in order to account for the plasmon dispersions in heavy alkali metals. These studies improved the agreement with the experimental results, but did not reproduce the shape of the dispersion curve in the all $q$ range where the experiments were performed. On the other hand, some researchers $[9,10]$ pointed out that interband transitions to the unoccupied $d$ states substantially modify the plasmon dispersions in heavy alkali metals (the socalled "band structure effect"). Aryasetiawan and Karlsson [9] performed ab initio calculations of the plasmon dispersions in solid alkali metals ( $\mathrm{Na}, \mathrm{K}, \mathrm{Rb}$, and $\mathrm{Cs}$ ) to investigate how the band structure effect influences the plasmon behaviors. These authors showed that the band structure effect becomes increasingly as important as going from $\mathrm{Na}$ to $\mathrm{Cs}$, and found that the band structure effect causes a very small dispersion in solid $\mathrm{Rb}$ and a negative dispersion in solid Cs. These results qualitatively reproduced the experimental trends, except for the detailed features such as the cusplike dispersion in solid Rb.

Theoretical studies on the electronic density of states (DOS) have given the information on the unoccupied $d$ states of heavy alkali metals both in the solid [11,12] and liquid [12-14]

*kazuhiro-matsuda@scphys.kyoto-u.ac.jp states. According to Jank et al. [12], it was found that the unoccupied $d$ states of $\mathrm{Rb}$ and $\mathrm{Cs}$ substantially change upon melting. Therefore, comparison of the plasmon behaviors in the solid state with those in the liquid state will give us valuable information on the band structure effect in heavy alkali metals. Up to now, plasmons in liquid alkali metals have been studied only for the light element [15]. The present work is an attempt to investigate the plasmon behaviors of the heavy alkali metal in the liquid state.

In this study, we carried out inelastic x-ray scattering (IXS) measurements for solid and liquid Rb. IXS technique has an advantage in that the spectra are purely composed of single scattering events, while EELS spectra in the high $q$ region suffer from multiple scattering events. In early IXS experiments, the small cross section limited the measurements to the light elements [16-19]. In recent years, however, with the advent of synchrotron radiation sources, IXS has become a standard tool to probe the dynamic response of electrons in materials including the heavier elements [20-22]. Moreover, unlike EELS, IXS measurements can be done even for liquid samples [15,23,24].

In this paper, we report the plasmon dispersions in solid and liquid $\mathrm{Rb}$. Upon melting, the plasmon energies increase in the range $q \geqslant 0.60 \AA^{-1}$, and the cusplike behavior is no longer observed for liquid $\mathrm{Rb}$. From a comparison of the unoccupied $d$ states in the DOS for solid $\mathrm{Rb}$ with those for liquid $\mathrm{Rb}$, we interpret that the increase of the plasmon energy originates from the reduction in the band structure effect upon melting. We also determine the $q$ dependence of the plasmon linewidth $\Delta E_{1 / 2}(q)$ and find that $\Delta E_{1 / 2}(q)$ in liquid $\mathrm{Rb}$ tends to be smaller than in solid $\mathrm{Rb}$ near $q=0$. We interpret that this behavior reflects the reduction in the effect of interband transitions on the plasmon damping upon melting, which is supported by the theoretical evaluation of $\Delta E_{1 / 2}(q=0)$ for both solid and liquid $\mathrm{Rb}$. Our results suggest that the effects of the unoccupied $d$ states on the plasmon energy and those of interband transitions on the linewidth, which are not considered in the electron gas model, become less important upon melting. These results indicate that the conduction electrons in $\mathrm{Rb}$ are better described with the electron gas model in the liquid state than in the solid state. 


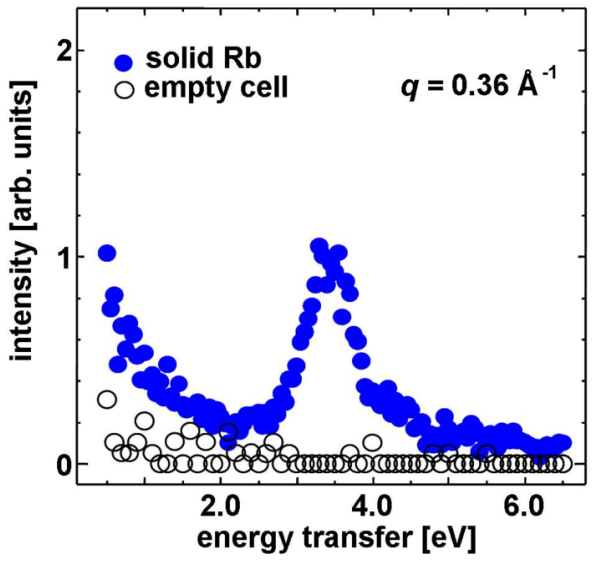

FIG. 1. (Color online) IXS spectra of solid $\mathrm{Rb}$ and the empty cell at $q=0.36 \AA^{-1}$ normalized by the incident beam intensity.

\section{EXPERIMENT}

IXS measurements were carried out on the Taiwan inelastic $\mathrm{X}$-ray scattering beam line BL12XU at SPring-8, designed for the studies on various types of electronic excitations [25]. The incident beam from the undulator was first monochromatized by a $\mathrm{Si}(111)$ double crystal monochromator and then by a $\mathrm{Si}(400)$ four-crystal high-resolution monochromator. IXS signals were collected with a diced, spherical, crystal analyzer and a $\mathrm{Si}$ detector in a near backscattering geometry. The scattered photon energy was fixed at $13835.3 \mathrm{eV}\left(=E_{0}\right)$, while incident photon energy was scanned by $6 \mathrm{eV}$ near $E_{0}$. The energy resolution, which is determined by the full width at half maximum (FWHM) of the quasielastic peak, was $0.22 \mathrm{eV}$. The $q$ resolution is determined by the opening of a mask $(85 \mathrm{~mm}$ diameter) in front of the analyzer. We evaluate it to be $0.25 \AA^{-1}$.

The measurements were performed at room temperature for solid (polycrystalline) $\mathrm{Rb}$ and at $333 \mathrm{~K}$ for liquid $\mathrm{Rb}$. (The melting temperature of $\mathrm{Rb}$ is $312 \mathrm{~K}$.) We used a thin-walled $(100 \mu \mathrm{m})$ sample cell made of sapphire to perform stable measurements for the liquid phase. The thickness of the sample was $300 \mu \mathrm{m}$. The sample was sealed under a He atmosphere to prevent oxidation. We heated the sample with a $\mathrm{Fe}-\mathrm{Cr}$ electronic resistant heater and monitored the temperature by alumel-chromel thermocouples. The range of the energy transfer was from 0.5 to $6.5 \mathrm{eV}$. The range of the momentum transfer was from 0.18 to $0.90 \AA^{-1}$ for solid $\mathrm{Rb}$ and from 0.18 to $0.80 \AA^{-1}$ for liquid $\mathrm{Rb}$. The measurements took three hours for each momentum transfer. We also measured the IXS from the empty cell and confirmed that the inelastic signals from the empty cell were negligible compared with those from the sample, as can be seen from Fig. 1 .

\section{RESULTS}

Figure 2 shows the IXS spectra of solid and liquid Rb as a function of the energy transfer $\hbar \omega$. The peak arising from plasmon is clearly observed around $\hbar \omega \sim 3.5 \mathrm{eV}$ both in solid and liquid $\mathrm{Rb}$ for each of the momentum transfers $q$. The IXS spectra were fitted by the sum of two Lorentzians [26], one representing the inelastic (plasmon), and the other the quasielastic contribution. The fitting functions were

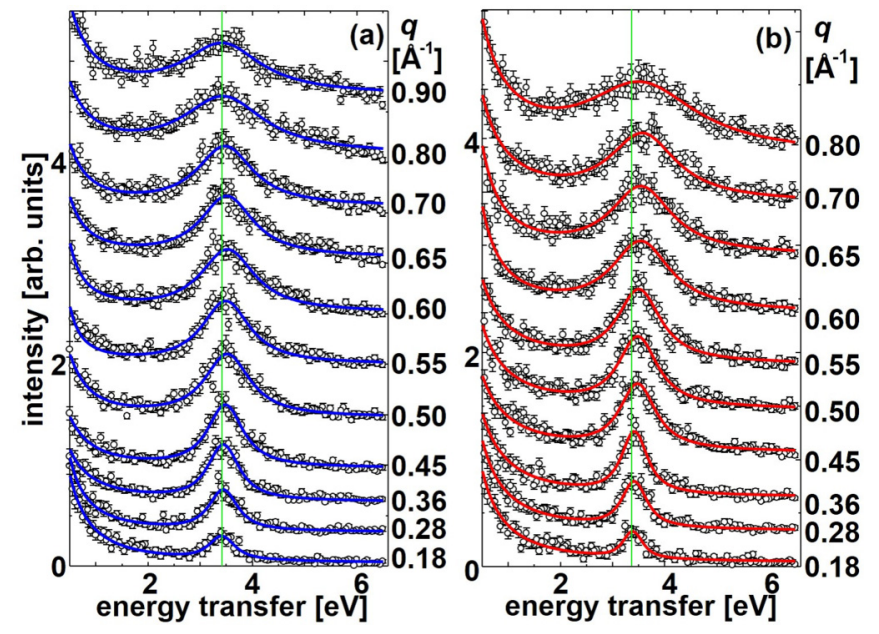

FIG. 2. (Color online) IXS spectra of (a) solid Rb and (b) liquid $\mathrm{Rb}$ normalized by the incident-beam intensity. Values at the right side of the figures are momentum transfers. Solid curves are the fitting functions. Vertical line denotes the peak position of the spectrum at $q=0.18 \AA^{-1}$.

convoluted with a Gaussian of $0.22 \mathrm{eV} \mathrm{FWHM}$, corresponding to the experimental energy resolution. These functions are indicated with solid curves in Fig. 2. The vertical line in each of the figures denotes the inelastic peak position in the spectrum at $q=0.18 \AA^{-1}$. Note that the shift of the peak position from the vertical line with increasing $q$ is larger in liquid $\mathrm{Rb}$ than in solid $\mathrm{Rb}$ beyond $q \sim 0.60 \AA^{-1}$.

Figure 3(a) shows the $q$ dependence of the plasmon energy determined by the fitting procedure. We can see the cusplike shape of the dispersion curve for solid $\mathrm{Rb}$, where, with increasing $q$, the plasmon energy gradually increases within the range $0.18 \AA^{-1} \leqslant q \leqslant 0.50 \AA^{-1}$ and decreases within the range $0.60 \AA^{-1} \leqslant q \leqslant 0.90 \AA^{-1}$. This behavior agrees with the results of the EELS measurements [1]. For liquid Rb, on the other hand, the plasmon energy monotonically increases with $q$ and it becomes larger than that of solid $\mathrm{Rb}$ in the range $q \geqslant 0.60 \AA^{-1}$.

The dotted line in Fig. 3(a) shows the boundary of the particle-hole ( $\mathrm{p}-\mathrm{h}$ ) continuum, which was estimated using the following equations based on the non-interacting electron gas model:

$$
\hbar \omega=\frac{|\boldsymbol{k}+\boldsymbol{q}|^{2}}{2 m}-\frac{|\boldsymbol{k}|^{2}}{2 m}, \quad|\boldsymbol{k}|<k_{\mathrm{F}}, \quad|\boldsymbol{k}+\boldsymbol{q}|>k_{\mathrm{F}},
$$

where $k_{\mathrm{F}}$ is the Fermi wave vector, $\boldsymbol{k}$ is the wave vector of an electron, and $m$ is the electron mass. The value of $k_{\mathrm{F}}$ used here is $k_{\mathrm{F}}=0.68 \AA^{-1}$, which is derived from the density of solid $\mathrm{Rb}$ at room temperature, $1.53 \mathrm{~g} \mathrm{~cm}^{-3}$. The experimental plasmon dispersions intersect the boundary of the $\mathrm{p}-\mathrm{h}$ continuum around $q \sim 0.50 \AA^{-1}$. Thus the plasmon cut-off wave vector $q_{\mathrm{c}}^{\text {expt }}$ is estimated to be $q_{\mathrm{c}}^{\text {expt }} \sim 0.50 \AA^{-1}$, above which a plasmon decays into a $\mathrm{p}-\mathrm{h}$ pair.

Figure 3(b) shows the $q$ dependence of the plasmon linewidth $\Delta E_{1 / 2}(q)$, determined from the FWHM of the Lorentzian curve. The values of $\Delta E_{1 / 2}(q)$ for liquid $\mathrm{Rb}$ tend to be smaller in $q<q_{\mathrm{c}}^{\text {expt }}$ and larger in $q>q_{\mathrm{c}}^{\text {expt }}$ than those of solid Rb. This behavior is seen more clearly in the inset of 


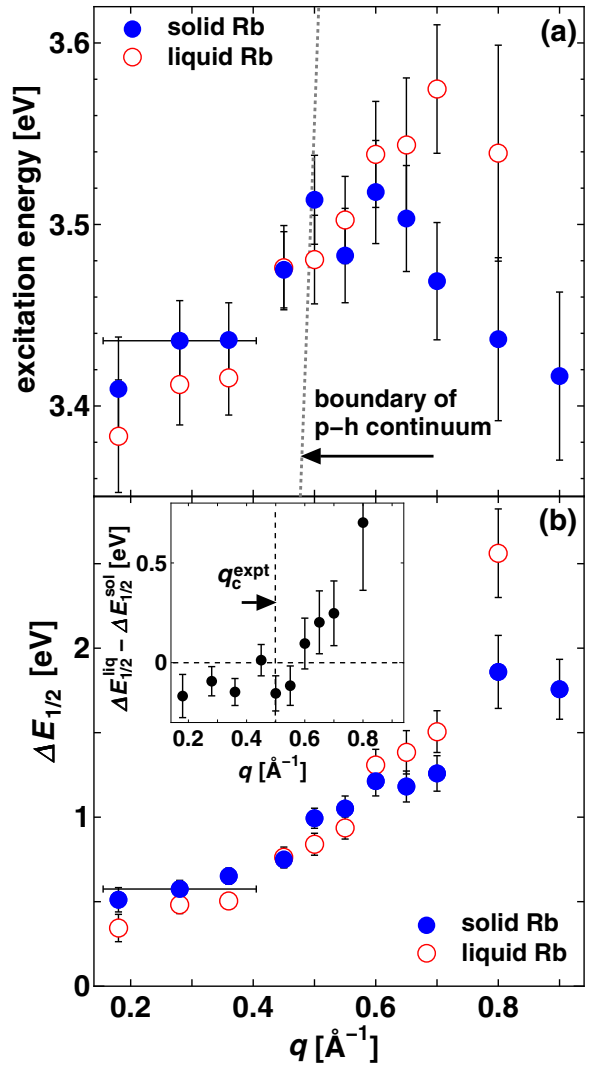

FIG. 3. (Color online) (a) Plasmon dispersions of solid and liquid $\mathrm{Rb}$. The boundary of the $\mathrm{p}-\mathrm{h}$ continuum is indicated by the dotted line. (b) $q$ dependence of the plasmon linewidth $\Delta E_{1 / 2}(q)$ of solid and liquid $\mathrm{Rb}$. The inset shows the difference of $\Delta E_{1 / 2}(q)$ between solid and liquid $\mathrm{Rb}$, which was derived by subtracting $\Delta E_{1 / 2}(q)$ of solid $\mathrm{Rb}$ from that of liquid $\mathrm{Rb}\left(\Delta E_{1 / 2}^{\mathrm{liq}}-\Delta E_{1 / 2}^{\mathrm{sol}}\right)$. The $q$ resolution $0.25 \AA^{-1}$ is indicated by the error bars for $q$ in both figures.

Fig. 3(b), which shows the difference of $\Delta E_{1 / 2}(q)$ between solid and liquid $\mathrm{Rb}$.

\section{DISCUSSION}

\section{A. Plasmon energy}

As mentioned in Sec. I, it has been shown that the plasmon energy in solid $\mathrm{Rb}$ is strongly modified by the interband transitions to unoccupied $d$ states [9]. Therefore, we discuss the unoccupied $\mathrm{DOS}$ of $\mathrm{Rb}$ to understand the variation in the plasmon dispersion upon melting.

Figure 4 shows the theoretical DOS for solid [11] and liquid [13] Rb. The DOS of solid Rb shown here is the result of full-potential linear muffin-tin orbital (LMTO) calculations for $300 \mathrm{~K}$ performed by Söderlind and Ross [11]. The DOS of liquid $\mathrm{Rb}$ was obtained from the Schommers-LMTO-recursion method [27-30] for $373 \mathrm{~K}$, which was carried out by Mirzoev et al. [13]. The DOS of solid Rb above the Fermi energy considerably deviates from the DOS of free electrons. Peaks are observed at about $1,2.5,4$, and $5 \mathrm{eV}$ in the DOS of solid $\mathrm{Rb}$, mainly because of the $d$-state contribution. Upon melting, the peaks at about 4 and $5 \mathrm{eV}$, observed in the DOS of solid Rb, are smoothed out. In addition, the broad peak at about $1.7 \mathrm{eV}$

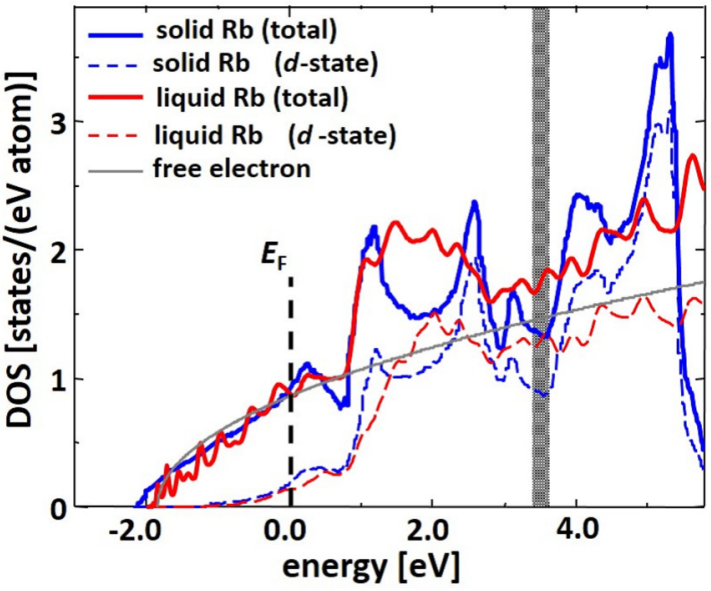

FIG. 4. (Color online) Theoretical DOS of solid (300 K) [11] and liquid (373 K) [13] Rb. Solid and dashed lines show the total DOS and the contribution from $d$ states, respectively. The thin solid line shows the DOS of free electrons corresponding to the electron density of $\mathrm{Rb}$. A shaded area shows the energy range where plasmon peaks were observed in the IXS measurements. The Fermi energy $E_{\mathrm{F}}$ is set to 0 .

appears for the liquid state, instead of the sharp features at about 1 and $2.5 \mathrm{eV}$ in the DOS of solid Rb.

Peaks in DOS modify the plasmon energy for the following reason. When unoccupied DOS has a peak at a certain energy, interband transitions to that energy become strong, which makes a maximum in the imaginary part of the dielectric function, $\varepsilon_{2}(\boldsymbol{q}, \omega)$. The $\varepsilon_{2}(\boldsymbol{q}, \omega)$ is generally associated with the real part of the dielectric function, $\varepsilon_{1}(\boldsymbol{q}, \omega)$, through the Kramers-Kronig relation,

$$
\varepsilon_{1}(\boldsymbol{q}, \omega)=1+\frac{2}{\pi} \int_{0}^{\infty} d \omega^{\prime} \frac{\omega^{\prime} \varepsilon_{2}\left(\boldsymbol{q}, \omega^{\prime}\right)}{\omega^{\prime 2}-\omega^{2}} .
$$

Therefore, the variation in $\varepsilon_{2}(\boldsymbol{q}, \omega)$ due to interband transitions modifies the plasmon energy, which is determined by $\varepsilon_{1}(\boldsymbol{q}, \omega)=0$.

Aryasetiawan et al. [9] discussed how interband transitions change the plasmon energy. According to their discussion, plasmon energy is lowered owing to the enhancement of interband transitions above the plasmon energy. To explain this lowering, they represented the last term in Eq. (2) as the sum of two terms with different integration ranges, $\left[0, \omega_{\mathrm{p}}^{0}\right]$ and $\left[\omega_{\mathrm{p}}^{0}, \infty\right]$. Here, $\omega_{\mathrm{p}}^{0}$ is the plasma frequency obtained without considering the enhancement of the interband transitions. Then, we can see that the term with the integration range $\left[\omega_{\mathrm{p}}^{0}, \infty\right]$ contributes positively to $\varepsilon_{1}(\boldsymbol{q}, \omega)$ at $\omega=\omega_{\mathrm{p}}^{0}$. Thus, when interband transitions are enhanced and $\varepsilon_{2}\left(\boldsymbol{q}, \omega^{\prime}\right)$ increases within the range $\omega^{\prime}>\omega_{\mathrm{p}}^{0}$, the value of $\varepsilon_{1}(\boldsymbol{q}, \omega)$ becomes larger at $\omega=\omega_{\mathrm{p}}^{0}$. The increase in $\varepsilon_{1}\left(\boldsymbol{q}, \omega=\omega_{\mathrm{p}}^{0}\right)$ results in the lowering of the plasmon energy relative to $\omega_{\mathrm{p}}^{0}$ [9], because the slope of $\varepsilon_{1}$ is generally positive at the plasmon energy.

For liquid Rb, peaks are not clearly seen in the DOS above the plasmon peak positions (shaded area in Fig. 4). On the other hand, for solid Rb, the peaks due to $d$ states are seen in the DOS at about 4 and $5 \mathrm{eV}$, the energies higher than the shaded energy range in Fig. 4. Therefore, from the above consideration, the 
TABLE I. Experimental values of the plasmon energy at $q=0$ $\left[\hbar \omega_{\mathrm{p}}(0)\right]$ and the plasmon dispersion coefficient $\alpha$.

\begin{tabular}{lcc}
\hline \hline & Solid Rb & Liquid Rb \\
\hline$\hbar \omega_{\mathrm{p}}(0)(\mathrm{eV})$ & $3.39 \pm 0.02$ & $3.37 \pm 0.02$ \\
$\alpha$ & $0.06 \pm 0.02$ & $0.06 \pm 0.02$
\end{tabular}

plasmon energy in solid $\mathrm{Rb}$ should be lowered by the interband transitions more strongly than in liquid $\mathrm{Rb}$ for the $q$ at which the $s-d$ transitions are allowed and the momentum conservation is satisfied. It should be stressed that the probability of the $s-d$ transitions increases with $q^{4}$ [9]. Thus, the lowering of the plasmon energy for solid $\mathrm{Rb}$ due to the interband transitions should be more evident for larger $q$. This is consistent with the observation that the plasmon energy of solid $\mathrm{Rb}$ is lower than that of liquid $\mathrm{Rb}$ for the large $q$ region [Fig. 3(a)].

In contrast to the DOS peaks above the plasmon energy $\omega_{\mathrm{p}}$, the $d$ states at 1 and $2.5 \mathrm{eV}$ in solid $\mathrm{Rb}$ are not expected to contribute significantly to $\omega_{\mathrm{p}}$ for the following reason. For the plasmon with positive dispersion, the difference between the excitation energy and the positions of the DOS peaks below $\omega_{\mathrm{p}}$ increases with $q$. This behavior indicates that the influence of such DOS features on $\omega_{\mathrm{p}}$ is relatively large for small $q$ range. On the other hand, the probability of $s-d$ transitions becomes smaller with decreasing $q$, which makes the effect of the $d$ states below $\omega_{\mathrm{p}}$ less significant. This is also true for liquid $\mathrm{Rb}$.

As shown in Fig. 3(a), the plasmon energies in solid $\mathrm{Rb}$ agree well with those in liquid $\mathrm{Rb}$ for $q \leqslant 0.50 \AA^{-1}$, which suggests that the band structure effect is not important in this $q$ range. To compare the plasmon dispersions in both states for $q \leqslant 0.50 \AA^{-1}$ in more detail, we fitted the function $\hbar \omega_{\mathrm{p}}(q)=\hbar \omega_{\mathrm{p}}(0)\left[1+\alpha\left(\hbar^{2} / m\right) q^{2}\right]$ to the dispersion curve in the range $q \leqslant 0.50\left(\sim q_{\mathrm{c}}^{\text {expt }}\right)$. Table I shows the results of the fitting. We find that the value of $\alpha$ for solid $\mathrm{Rb}$ agrees with that for liquid $\mathrm{Rb}$. A slightly lower value of $\hbar \omega_{\mathrm{p}}(0)$ for liquid $\mathrm{Rb}$ is explained by the reduction in the electron density upon melting as follows. The $\hbar \omega_{\mathrm{p}}(0)$ is given by the relation $\hbar \omega_{\mathrm{p}}(0)=\hbar \sqrt{4 \pi n e^{2} / m \varepsilon_{\mathrm{B}}}, \varepsilon_{\mathrm{B}}=1+4 \pi n_{\mathrm{i}} \chi_{\mathrm{i}}$, where $n$ is the electron density, $n_{\mathrm{i}}$ is the ionic density, and $\chi_{\mathrm{i}}$ is the ionic polarizability. We set $\chi_{\mathrm{i}}=1.5 \times 10^{23} \mathrm{~cm}^{3}$, the value estimated by Mayer et al. [31]. The density of $\mathrm{Rb}$ reduces from 1.53 to $1.46 \mathrm{~g} \mathrm{~cm}^{-3}$ upon melting. From these values, $\hbar \omega_{\mathrm{p}}(0)$ is estimated to be $3.52 \mathrm{eV}$ for solid $\mathrm{Rb}$ and $3.47 \mathrm{eV}$ for liquid $\mathrm{Rb}$. According to this estimation, the ratio of the value of $\hbar \omega_{\mathrm{p}}(0)$ for liquid $\mathrm{Rb}$ to that for solid $\mathrm{Rb}$ is 0.986 , which reasonably explains the experimental value $0.994 \pm 0.008$.

\section{B. Plasmon linewidth}

As seen from the inset of Fig. 3(b), the linewidth $\Delta E_{1 / 2}(q)$ of liquid $\mathrm{Rb}$ tends to be larger than that of solid $\mathrm{Rb}$ in the range $q>q_{\mathrm{c}}^{\text {expt }}\left(\sim 0.5 \AA^{-1}\right)$. Considering the fact that a plasmon decays into a p-h pair beyond $q_{\mathrm{c}}^{\text {expt }}$, the observed tendency suggests that the decay into a $\mathrm{p}$-h pair is stronger in liquid $\mathrm{Rb}$ than in solid $\mathrm{Rb}$. Such an enhanced damping can be explained by the increase in the unoccupied DOS upon melting in the energy range where the plasmons are observed (shaded area in Fig. 4).

On the other hand, careful analysis is required to understand the fact that the $\Delta E_{1 / 2}(q)$ tends to decrease upon melting for $q<q_{\mathrm{c}}^{\text {expt }}$. To understand this behavior, we calculated the linewidth at $q=0$ for both solid and liquid $\mathrm{Rb}$ [32]. In the calculation, two effects are considered, i.e., interband transitions induced by the ionic potential and phonon-assisted intraband (or interband) transitions [33,34]. If we neglect the interference between these effects, the linewidth can be written as the sum of their contributions:

$$
\Delta E_{1 / 2}(0)=\Delta E_{1 / 2}^{\mathrm{ion}}(0)+\Delta E_{1 / 2}^{\mathrm{ph}}(0),
$$

where we denote the two effects as "ion" (ionic potential) and "ph" (phonon assisted).

The linewidth at $q=0$ is calculated by the product of the plasma frequency and the imaginary part of the dielectric function [34], and thus $\Delta E_{1 / 2}^{\text {ion }}(0)$ can be written as follows:

$$
\Delta E_{1 / 2}^{\text {ion }}(0)=\hbar \omega_{\mathrm{p}} \varepsilon_{2}^{\text {ion }}\left(0, \omega_{\mathrm{p}}\right),
$$

where $\varepsilon_{2}^{\text {ion }}(q, \omega)$ is the imaginary part of the dielectric function including the effect of the ionic potential. For the solid state, $\varepsilon_{2}^{\text {ion }}(0, \omega)$ is represented by [34]

$$
\begin{aligned}
\varepsilon_{2}^{\text {ion }}\left(0, \omega_{\mathrm{p}}\right)= & \frac{4 n_{110}\left|v_{\mathrm{s}}\left(G_{110}\right) / E_{\mathrm{F}}\right|^{2}\left(G_{110} / k_{\mathrm{F}}\right)^{4}}{3\left(\hbar \omega_{\mathrm{p}} / E_{\mathrm{F}}\right)^{4}} \\
& \times \operatorname{Im} \varepsilon_{\mathrm{L}}\left(G_{110}, \omega_{\mathrm{p}}\right) \quad(\text { for solid })
\end{aligned}
$$

where $G_{110}$ is the reciprocal lattice vector along the [110] direction, $n_{110}$ is the number of the reciprocal lattice vectors equivalent to $G_{110}, v_{\mathrm{s}}\left(q^{\prime}\right)$ is the Fourier component of screened pseudopotential, and $\varepsilon_{\mathrm{L}}\left(q^{\prime}, \omega\right)$ is the Lindhard dielectric function [35].

To evaluate $\Delta E_{1 / 2}^{\text {ion }}(0)$ for the liquid state, we have derived the following expression [32]:

$$
\begin{aligned}
\varepsilon_{2}^{\text {ion }}\left(0, \omega_{\mathrm{p}}\right)= & \frac{4}{3 n_{\mathrm{i}}\left(\hbar \omega_{\mathrm{p}} / E_{\mathrm{F}}\right)^{4}} \int \frac{d^{3} q^{\prime}}{(2 \pi)^{3}}\left(\frac{q^{\prime}}{k_{\mathrm{F}}}\right)^{4} \\
& \times\left|\frac{v_{\mathrm{s}}\left(q^{\prime}\right)}{E_{\mathrm{F}}}\right|^{2} S\left(q^{\prime}\right) \operatorname{Im} \varepsilon_{\mathrm{L}}\left(q^{\prime}, \omega_{\mathrm{p}}\right)
\end{aligned}
$$

(for liquid)

where $S\left(q^{\prime}\right)$ is the liquid structure factor.

Through Eqs. (4) and (6), the effect of interband transitions on the plasmon damping is associated with the short range order of ions, which is characterized by $S\left(q^{\prime}\right)$. The relation between the short range order and the effect of interband transitions has been discussed in the previous IXS studies for liquid samples [15,24], but has not been described in a quantitative manner. Equation (6) enables us to evaluate quantitatively the effect of interband transitions on the linewidth using the information on the short range order of ions in liquid. The details of the calculation, including the phonon part, have been given in our previous work [32].

Figure 5 shows the experimentally determined linewidth in small $q$ region and the calculated linewidth at $q=0$. The experimental results are fitted by the quadratic function of $q$ as indicated by the dashed lines in Fig. 5. (The fitting range is from 0.18 to $0.45 \AA^{-1}$.) The calculated results quantitatively agree with the extrapolated values at $q=0$ for both solid and liquid $\mathrm{Rb}$. 


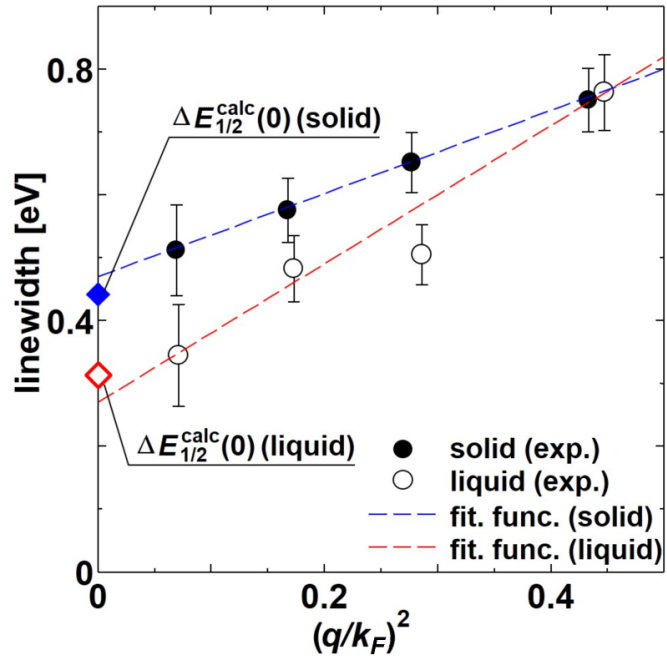

FIG. 5. (Color online) Plasmon linewidth of solid and liquid $\mathrm{Rb}$ in small $q$ region are plotted against $\left(q / k_{\mathrm{F}}\right)^{2}$, where $k_{\mathrm{F}}$ is the Fermi wave number. Closed circles show the experimental data for solid $\mathrm{Rb}$ and open circles for liquid Rb. Diamonds indicate the calculation for the solid and the liquid states at $q=0$. Dashed lines are the quadratic functions of $q$ fitted to the experimental data.

Table II shows the experimental and calculated [32] values of the linewidth for $q=0$. (Hereafter, we denote $\Delta E_{1 / 2}^{\text {expt }}(0)$ and $\Delta E_{1 / 2}^{\text {calc }}(0)$, respectively.) The value of $\Delta E_{1 / 2}^{\text {expt }}(0)$ was determined by the fitting with a quadratic function. In the table, the values of the two terms in Eq. (3), $\Delta E_{1 / 2}^{\mathrm{ion}}(0)$ and $\Delta E_{1 / 2}^{\mathrm{ph}}(0)$, are also indicated. As noticed from the table, the $\Delta E_{1 / 2}^{\mathrm{ph}}(0)$ of liquid $\mathrm{Rb}$ is larger than that of solid $\mathrm{Rb}$, indicating the increase of thermal motion of ions upon melting. Thus, the observation that the value of $\Delta E_{1 / 2}^{\text {calc }}(0)\left[=\Delta E_{1 / 2}^{\text {ion }}(0)+\Delta E_{1 / 2}^{\mathrm{ph}}(0)\right]$ in the liquid state is smaller than that in the solid state is attributed to the decrease in $\Delta E_{1 / 2}^{\text {ion }}(0)$ upon melting. Therefore, we interpret that the experimentally observed decrease in the linewidth upon melting is caused by the suppression of the effect of interband transitions accompanied by the vanishing of the crystalline structure.
TABLE II. Experimental value of the linewidth at $q=0$ $\left[\Delta E_{1 / 2}^{\text {expt }}(0)\right]$ and the calculated one $\left[\Delta E_{1 / 2}^{\text {calc }}(0)\right]$, which is a sum of the ionic part $\left[\Delta E_{1 / 2}^{\mathrm{ion}}(0)\right]$ and the phonon part $\left[\Delta E_{1 / 2}^{\mathrm{ph}}(0)\right]$. Calculated results are taken from our previous work [32]. All values are in $\mathrm{eV}$.

\begin{tabular}{lcccc}
\hline \hline & $\Delta E_{1 / 2}^{\mathrm{expt}}(0)$ & $\Delta E_{1 / 2}^{\mathrm{calc}}(0)$ & $\Delta E_{1 / 2}^{\mathrm{ion}}(0)$ & $\Delta E_{1 / 2}^{\mathrm{ph}}(0)$ \\
\hline Solid & $0.47 \pm 0.07$ & 0.44 & 0.42 & 0.022 \\
Liquid & $0.27 \pm 0.13$ & 0.31 & 0.27 & 0.043 \\
\hline \hline
\end{tabular}

\section{CONCLUSION}

We have performed IXS measurements of solid and liquid $\mathrm{Rb}$ to determine the $q$ dependence of the plasmon energy and the linewidth. The comparison of the plasmon energies in the solid state with those in the liquid state suggests that the band structure effect becomes less important upon melting. The theoretical analysis on $\Delta E_{1 / 2}(q)$ for both solid and liquid $\mathrm{Rb}$ reveals that the narrowing of $\Delta E_{1 / 2}(q)$ near $q=0$ upon melting reflects the decrease in the effect of interband transitions on the plasmon damping.

The observed features for the plasmon energy and the linewidth in the solid and the liquid states suggest that the conduction electrons in liquid $\mathrm{Rb}$ are more suitably described with the electron gas model than in the solid $\mathrm{Rb}$.

\section{ACKNOWLEDGMENTS}

The authors thank Dr. A. N. Sobolev for providing us the data of the DOS for liquid $\mathrm{Rb}$. This work was supported by Grant-in-Aid for Scientific Research Fund (Research No. 24540401 and No. 23241033) and the Global COE Program "The Next Generation of Physics, Spun from Universality and Emergence" from the Ministry of Education, Culture, Sports, Science and Technology of Japan. The synchrotron radiation experiments were performed at the SPring-8 with the approval of Japan Synchrotron Radiation Research Institute (Grants No. 2010B4260, No. 2011B4257, and No. 2012B4261) and the National Synchrotron Radiation Research Center (Grant No. 2010_2_009).
[1] A. vom Felde, J. Sprösser-Prou, and J. Fink, Phys. Rev. B 40, 10181 (1989).

[2] P. Vashishta and K. S. Singwi, Phys. Rev. B 6, 875 (1972).

[3] L. Serra, F. Garcias, M. Barranco, N. Barberín, and J. Navarro, Phys. Rev. B 44, 1492 (1991).

[4] G. Kalman, K. Kempa, and M. Minella, Phys. Rev. B 43, 14238 (1991).

[5] M. Taut and K. Sturm, Solid State Commun. 82, 295 (1992).

[6] M. Taut, J. Phys.: Condens. Matter 4, 9595 (1992).

[7] E. Lipparini, S. Stringari, and K. Takayanagi, J. Phys.: Condens. Matter 6, 2025 (1994).

[8] H. M. Böhm, S. Conti, and M. P. Tosi, J. Phys.: Condens. Matter 8, 781 (1996).

[9] F. Aryasetiawan and K. Karlsson, Phys. Rev. Lett. 73, 1679 (1994).

[10] A. Fleszar, R. Stumpf, and A. G. Eguiluz, Phys. Rev. B 55, 2068 (1997).
[11] P. Söderlind and M. Ross, J. Phys.: Condens. Matter 12, 921 (2000).

[12] W. Jank and J. Hafner, J. Phys.: Condens. Matter 3, 6947 (1991).

[13] A. A. Mirzoev, A. A. Mirzoev, Jr., A. N. Sobolev, and B. R. Gelchinski, J. Phys.: Condens. Matter 20, 114104 (2008).

[14] A. Vorontsov, A. A. Mirzoev, G. P. Vyatkin, and A. Sobolev, J. Non-Cryst. Solids 353, 3206 (2007).

[15] J. P. Hill, C. C. Kao, W. A. C. Caliebe, D. Gibbs, and J. B. Hastings, Phys. Rev. Lett. 77, 3665 (1996).

[16] W. Schülke, U. Berg, and O. Brümmer, Phys. Status Solidi B 35, 227 (1969).

[17] G. Priftis, Phys. Rev. B 2, 54 (1970).

[18] D. M. Miliotis, Phys. Rev. B 3, 701 (1971).

[19] P. Eisenberger, P. M. Platzman, and K. C. Pandy, Phys. Rev. Lett. 31, 311 (1973). 
[20] S. Huotari, C. Sternemann, M. C. Troparevsky, A. G. Eguiluz, M. Volmer, H. Sternemann, H. Muller, G. Monaco, and W. Schülke, Phys. Rev. B 80, 155107 (2009).

[21] S. Huotari, J. A. Soininen, G. Vankó, G. Monaco, and V. Olevano, Phys. Rev. B 82, 064514 (2010).

[22] H.-C. Weissker, J. Serrano, S. Huotari, E. Luppi, M. Cazzaniga, F. Bruneval, F. Sottile, G. Monaco, V. Olevano, and L. Reining, Phys. Rev. B 81, 085104 (2010).

[23] C. Sternemann, A. Kaprolat, and W. Schülke, Phys. Rev. B 57, 622 (1998).

[24] C. A. Burns, P. Abbamonte, E. D. Isaacs, and P. M. Platzman, Phys. Rev. Lett. 83, 2390 (1999).

[25] Y. Q. Cai, P. Chow, C. C. Chen, H. Ishii, K. L. Tsang, C. C. Kao, K. S. Liang, and C. T. Chen, in Synchrotron Radiation Instrumentation: Eighth International Conference, edited by
T. Warwick et al., AIP Conf. Proc. No. 705 (AIP, Melville, NY, 2004), p. 340.

[26] A. L. Fetter and J. D. Walecka, Quantum Theory of ManyParticle Systems (Dover, New York, 2003).

[27] W. Schommers, Phys. Rev. A 28, 3599 (1983).

[28] O. K. Andersen and O. Jepsen, Phys. Rev. Lett. 53, 2571 (1984).

[29] R. Haydock, Solid State Phys. 35, 215 (1980).

[30] H. J. Nowak, O. K. Andersen, T. Fujiwara, O. Jepsen, and P. Vargas, Phys. Rev. B 44, 3577 (1991).

[31] J. E. Mayer and M. G. Mayer, Phys. Rev. 43, 605 (1933).

[32] K. Kimura, K. Matsuda, and M. Yao, J. Phys. Soc. Jpn. 82, 115001 (2013).

[33] M. Hasegawa, J. Phys. Soc. Jpn. 31, 649 (1971).

[34] K. Sturm and L. E. Oliveira, Phys. Rev. B 24, 3054 (1981).

[35] J. Lindhard, K. Dan. Vidensk. Selsk., Mat.-Fys. Medd. 28, 3 (1954). 